\title{
CDU-nahe Lizenzzeitungen (VII): „Westfalenpost"
}

\author{
von Heinz-Dietrich Fischer
}

Nachdem das "Westfalen-Blatt" (Bielefeld) bereits am 15. März 1946 erschienen war1, sollte es mehr als einen Monat dauern, bis im westfälischen Bereich die CDU ein weiteres ihr nahestehendes Presseorgan seitens der britischen Besatzungsbehörde zugebilligt erhielt. Die CDU Westfalens hatte schon im Herbst 1945 „eine Pressekommission gebildet"2, welche die Zeitungswünsche der Partei nachhaltig bei den Briten vertreten sollte. Als besondere Sachkenner des Pressewesens waren von der Partei die Verleger Wilhelm Bitter (Recklinghausen), Lambert Lensing (Dortmund) und Otto Rippel (Hagen) in diese Kommission delegiert worden. ${ }^{3}$ Anders als im rheinischen Raum, wo es im Februar/März 1946 schon drei CDU-nahe Zeitungsgründungen gegeben hatte ${ }^{4}$, verhielten sich die britischen Lizenzgeber in Westfalen weitaus reservierter gegenüber den Zeitungswünschen der CDU. Ein immer wieder ins Feld geführter Grund für diese Erscheinung wird darin erblickt, daß die CDU in Westfalen sich heftigst mit der wiedergegründeten Zentrumspartei auseinandersetzte, welche schließlich auch zwei Zeitungen - „Neuer Westfälischer Kurier" (Werl), „Rhein-RuhrZeitung" (Essen) - erhielt, die von der CDU als ihr verlorengegangene Gründungen empfunden wurden.

Das zögernde Verhalten der Briten im westfälischen Bereich Nordrhein-Westfalens hatte jedoch auch einen Grund darin, daß potentielle Lizenzträger für CDU-nahe Zeitungen von der Besatzungsmacht nicht als solche akzeptiert wurden. Dies galt besonders für den Mitbegründer der CDU Westfalens und früheren Herausgeber des Dortmunder Zentrumsblattes „Tremonia“, Lambert Lensing, der von den Briten als Träger einer Zeitungslizenz abgelehnt wurde. Gegen ihn waren verschiedene Anschuldigungen erhoben worden, in der Zeit vor 1945 seine Zeitung "Tremonia" in den Dienst des Nationalsozialismus gestellt zu haben. Zwar setzten sich der westfälische CDU-Vorsitzende Johannes Gronowski und rund 20 weitere Persönlichkeiten bei den Briten für eine politische Rehabilitierung Lensings ein, erzielten damit jedoch keinen Erfolg. ${ }^{5} \mathrm{Als}$ die Briten dann schließlich eine zweite CDU-orientierte Zeitung für Westfalen genehmigten, wurden andere Persönlichkeiten aus Kreisen der Partei für die Leitung des neuen Blattes genommen, und auch der Erscheiungsort des neuen Organs wurde nicht nach Dortmund gelegt, wo die Druckerei Lensings zur Verfügung stand. Am 26. April 1946 erschien in Soest die erste Ausgabe des CDU-Richtungsblattes "Westfalenpost". Zum Zeitpunkt des Entstehens der Zeitung war Soest der Wohnsitz des Lizenzträgers Dr. Artur Sträter, der bis zu seiner Berufung in das Amt des Justizministers des Landes Nordrhein-Westfalen im Dezember 1946 als Rechtsanwalt und Notar in Soest amtierte. ${ }^{6}$ Zum Geschäftsführer der "Westfalenpost“" wurde der ehemalige preußische Kultusminister Dr. Otto Boelitz bestellt ${ }^{7}$, der später eine maßgebliche Rolle beim Auf- und Ausbau des „Verein Union-Presse“ spielen sollte. ${ }^{8}$

Dr. Heinz Dietrich Fischer ist Wiss. Assistent an der Sektion für Publizistik und Kommunikation der Ruhr-Universität Bochum. 
Die „Westfalenpost", welche seit ihrer ersten Nummer den Untertitel „Für demokratischen Aufbau im christlichen Geist“ führte, enthält in ihrer ersten Ausgabe vom 26. April $1946 \mathrm{zwei}$ als programmatische Außerungen zu wertende Beiträge. In dem von Dr. Artur Sträter bereits unter dem Datum des 15. April verfaßten Geleitwort heißt es über Ziele und Aufgaben der "Westfalenpost" ${ }^{\text {"⿻ }: ~ „ E i n e ~ k l a r ~ a u s g e r i c h t e t e ~ u n d ~}$ zielbewußt geleitete Zeitung hat heute beim Wiederaufbau Deutschlands und bei der Sammlung der Geister eine ausschlaggebende Bedeutung ... Dieses große Ziel ist für unsere Zeitung, die heute zum ersten Male erscheint, richtunggebend. Sie trägt in ihrem Kopf den Untertitel ,Für demokratischen Aufbau im christlichen Geist'. Damit bekennt sie sich mit ihrem ganzen Mitarbeiterstab zu den ewigen Lebenskräften und Grundwahrheiten des Christentums, die sie in ihrem Dienst an der Gemeinschaft klar verdeutlichen will. Diese Kräfte wirksam werden zu lassen und sie nicht nur in unser privates, sondern auch in das politische und kulturelle Leben einfluten zu lassen, wird unser aller Bemühen sein. Wir sind uns der hohen Verantwortung bewußt, die wir mit Übernahme der Zulassung dieser Zeitung sowohl gegenüber den Besatzungsbehörden wie gegenüber dem deutschen Volke übernommen haben. Wir können diese Verantwortung nur tragen, wenn wir einerseits das uns anvertraute Werkzeug ganz in den Dienst der Wahrheit stellen, und andererseits sorgfältig aufhorchen auf die Stimme unserer Leser ....".

In einem anderen, ungezeichneten Beitrag wurde noch konkreter auf die publizistische Aufgabe der „Westfalenpost" hingewiesen, wenn es dort $u$. a. hieß ${ }^{10}$ : „Möge der Weg, den sie [die Zeitung] heute antritt, für alle, die mit ihr schreiten wollen, ein Weg aus dem Chaos werden, in das die ,Revolution des Nihilismus' unser Volk zu stürzen vermochte! Mut und Wille zur Wahrheit und zur Gerechtigkeit sollen die Feder der ,Westfalenpost' führen ... Um die Revolution des Nihilismus zu überwinden, bedarf es einer entschiedenen Absage an den Ungeist der Zerstörung und Verneinung ... Die ,Westfalenpost' erklärt daher am Anfang ihres Weges allen, die ihr begegnen, daß sie entschlossen ist, aus Uberzeugung und Verantwortung zum Manifest des hohen Ethos $\mathrm{zu}$ stehen, wie es in der Bergpredigt des Nazareners mit einmaliger Gültigkeit formuliert worden ist ... Mit ihrem Bekenntnis zu Christentum und Demokratie hat die ,Westfalenpost' grundlegend ausgesagt, was der Inhalt ihres lebendigen Dienstes an Volk und Heimat sein und bleiben soll. Sie will eine Heimatzeitung westfälischer Menschen werden, ihre treue Begleiterin auf dem Weg aus Chaos und Not zum Gemeinschaftswerk des Aufbaues einer neuen staatlichen und sozialen Ordnung. Sie betritt ihren Weg in der zuversichtlichen Hoffnung, daß der Beweis des guten Willens, den wir - die Zeitung und ihre Leser - jederzeit führen wollen, uns helfen wird, endlich das gemeinsame Band zu finden, das die Völker Europas eint...".

Mit diesen publizistischen Zielsetzungen begann die Zeitung ihr Erscheinen, deren Verlagsort zwar Soest war, die jedoch auf den Druckanlagen des Verlages Lensing in Dortmund technisch hergestellt wurde. Nur dort schien die erforderliche maschinelle Apparatur vorhanden zu sein, die Herstellung einer Auflage von 270000 Exemplaren (dienstags und freitags) zu gewährleisten. Das Verbreitungsgebiet der "Westfalenpost “ war von der lizenzgebenden Besatzungsbehörde auf den Regierungsbezirk Arnsberg sowie auf den südwestlichen Teil des Regierungsbezirks Münster festgelegt worden. Die Satzherstellung erfolgte an nicht weniger als elf Orten des Verbreitungsgebietes. Auswärtige Redaktionen wurden nicht nur in den Kreisstädten des Verbreitungsraumes unterhalten, sondern auch in Düsseldorf, Frankfurt a. M. und München. Ein gewisser überregionaler Anspruch wurde mit der besonderen Einrichtung einer speziellen überlokalen Ausgabe für den allgemeinen Versand über den engeren Erscheinungs- 
radius hinaus bekundet. Neben dieser Hauptausgabe gab es folgende - zumeist lokal begrenzte - Ausgaben: $\mathrm{A}=$ Soest, $\mathrm{B}=$ Lippstadt, $\mathrm{C}=$ Brilon, $\mathrm{D}=$ Meschede, $\mathrm{E}=$ Arnsberg, $\mathrm{F}=$ Neheim-Hüsten, $\mathrm{G}=$ Olpe, $\mathrm{H}=$ Siegen, $\mathrm{I}=$ Berleburg, $\mathrm{K}=$ Iserlohn, $\mathrm{L}=$ Lüdenscheid, $\mathrm{M}=$ Altena, $\mathrm{N}=$ Witten, $\mathrm{O}=\mathrm{Hamm}$, $\mathrm{P}=$ Lünen/Witten, $\mathrm{Q}=$ Dortmund, $\mathrm{R}=$ Hagen, $\mathrm{S}=$ Hattingen, $\mathrm{T}=$ Herne/Castrop-Rauxel, $U=$ Bochum, $\mathrm{V}=$ Wattenscheid/Wanne-Eickel, $\mathrm{W}=$ Gelsenkirchen, $X=$ Bottrop/Gladbeck, $Y=$ Recklinghausen-Stadt und $Z=$ Recklinghausen-Land. Bemerkenswert ist die Tatsache, daß mehrere dieser Ausgaben, insbesondere jene aus dem Dortmunder Raum, die Zusatzbezeichnung "Ruhr-Nachrichten " führten" ${ }^{11}$, ein Zeitungstitel, der ab 1949 in Dortmund von Lambert Lensing für eine Zeitung seines Hauses übernommen wurde ${ }^{12}$, welche seit dem 1. März 1949 erschien. Von diesem Zeitpunkt ab verlor die "Westfalenpost" Teile ihres in das Industriegebiet hineinragenden Absatzgebietes und konzentrierte sich fortan im wesentlichen auf Gebiete des Sauer- und Siegerlandes. Der veränderten Situation Rechnung tragend, wurde der Verlagssitz der „Westfalenpost “ am 30. April 1950 von Soest nach Hagen i. W. verlegt. ${ }^{13}$ Schon vorher war das Blatt nicht nur in Dortmund, sondern auch in Hamm und Hagen gedruckt worden, denn nur durch die Herstellung an mehreren Druckorten war es möglich, das relativ große Verbreitungsgebiet des Morgens zeitgleich mit den 17 Nebenausgaben des Blattes zu versorgen. ${ }^{14}$ Im Zeitraum 1950/51, als die "Westfalenpost" eine Gesamtauflage von 156000 täglichen Exemplaren aufwies, wurde die Zeitung an den drei Druckorten Hagen, Hamm und Siegen hergestellt. Neben Artur Sträter, den ursprünglichen Lizenzinhaber, waren zu diesem Zeitpunkt Josef Schrage und Otto Boelitz als Mitherausgeber getreten. ${ }^{15}$

Im 1. Vierteljahr 1953 betrug die Auflage nur noch rund 116500 Stück, die sich auf eine Haupt- und 24 Nebenausgaben verteilte und zu 99 Prozent im Abonnement vertrieben wurde. ${ }^{16}$ Der von der Zeitung seit ihrer Begründung im Jahre 1946 geführte Untertitel „Für demokratischen Aufbau im christlichen Geist" erschien zum letzten Male in der Ausgabe vom 6. Mai 1955. „Maßgebend für den Verzicht auf diese Zeile war die Tatsache", heißt es in einer Verlagsäußerung, „daß am 5. Mai 1955 mit der Hinterlegung der Ratifikationsurkunden zum Deutschland- und zum Truppenvertrag das Besatzungsregime endete und die Bundesrepublik ein souveräner Staat wurde. Damit konnte die Phase des, demokratischen Aufbaus' während der ersten Nachkriegsjahre als beendet gelten." 17 Dem schon 1946 formulierten Ziel entsprechend, sich eines "lebendigen Dienstes an Volk und Heimat" zu befleißigen, wurde später der neue Untertitel „Stimme der Heimat - Echo der Welt ${ }^{\alpha}$ gewählt. ${ }^{18}$

Um die publizistische und wirtschaftliche Position der "Westfalenpost" zu festigen, wurde zu Beginn des Jahres 1958 eine Redaktions- und Anzeigengemeinschaft mit den "Ruhr-Nachrichten“ (Dortmund) eingerichtet, ohne daß die „Westfalenpost " künftig den gesamten Mantel von der Dortmunder Zeitung übernahm. Vielmehr wurde der redaktionelle Einfluß des Herausgebers der "Westfalenpost" vertraglich gesichert, und darüber hinaus blieben die Verlage der "Ruhr-Nachrichten“ wie der „Westfalenpost “ wirtschaftlich völlig selbständige Unternehmen. ${ }^{19}$ In einer gemeinsamen Erklärung informierten die Verleger beider Zeitungen ihre Leserschaft über den vollzogenen Schritt der Kooperation mit folgenden Worten ${ }^{20}$ : „Mit der heutigen Ausgabe treten $z$ wei der größten christlich-bürgerlichen Zeitungsverlage Westdeutschlands in eine enge Arbeitsgemeinschaft ein. Die Herausgeber der ,Ruhr-Nachrichten' [Lambert Lensing] und der, Westfalenpost' [Dr. Artur Sträter] sind übereingekommen, für die über 300000 Abonnenten eine im Hauptteil für Redaktion und Anzeigen einheitlich gestaltete Tageszeitung zu schaffen. Dadurch soll mit den modernsten Mitteln aus 
einer bewußt christlichen Grundhaltung heraus die weiteste Offentlichkeit angesprochen werden. Die neue Arbeitsgemeinschaft $z$ wischen ,Ruhr-Nachrichten' und ,Westfalenpost' erstreckt sich auf den größten geschlossenen Wirtschafts- und Bevölkerungsraum der Bundesrepublik ... Eine große Zeitung muß drinnen wie draußen leistungsfähig sein. Die künftig gemeinsame Hauptredaktion der beiden Verlage lehnt eine billige Sensationsmache ebenso ab wie eine kautschukartige Meinung, die jedem nach dem Munde redet. Ober allem steht die Glaubwürdigkeit der Zeitung und das Vertrauen des Lesers...".

Mit dieser Kooperationsmaßnahme wurden beide Zeitungen zusammen die auflagestärkste Einheit unter den CDU/CSU-nahen Blättern, die im "Verein Union-Presse“ zusammengefaßt waren. Im 3. Quartal 1960 betrug die gemeinsame Auflage 360600 Exemplare. ${ }^{21}$ Acht Jahre später war die verbreitete Auflage auf 400964 angestiegen.22 Die "Westfalenpost" hat in ihrem Erscheinungsbereich festgestellt, daß die Bindung zur Leserschaft dermaßen eng ist, daß neun von zehn Lesern die "Westfalenpost" "sehr stark“, "stark“ bzw. „ein wenig vermissen“ würden, wenn das Blatt aus technischen Gründen einmal für einen längeren Zeitraum am Erscheinen gehindert sein würde. ${ }^{23}$ In der politischen Haltung der "Westfalenpost " hat sich keinerlei Anderung vollzogen, wenn man folgende aus dem Jahre 1964 stammende Eigentypisierung zitiert ${ }^{24}$ : „Die ,Westfalenpost' ist kein ,Sprachorgan' der CDU; sie bekennt sich zu einer Politik aus christlicher Verantwortung, wie sie auch die CDU im Grundsatz vertritt. Eine ,christliche Politik' gibt es nach unserer Auffassung nicht."

Anmerkungen:

1. Vgl. Heinz-Dietrich Fischer: „Westfalen-Blatt“, in: CS, 2. Jg. / Nr. 3 (1969), S. 230 ff.

2. Leo Schwering: Frühgeschichte der Christlich-Demokratischen Union, Recklinghausen 1963, S. 206.

3. Daselbst.

4. Es handelte sich um die „Aachener Volkszeitung“, die "Rheinische Post" sowie die „Kölnische Rundschau ${ }^{\alpha}$ (vgl. die Kurzmonographien hierüber in CS, Jg. 1969.)

5. Informationen aus Akten des Nachlasses Lambert Lensing, Dortmund.

6. Brief von Karl Peukert, Chef vom Dienst "Westfalenpost" (Hagen) an d. Verf. vom 30. Juni 1964, S. 1.

7. Handbuch Deutsche Presse, hrsgg. vom Nordwestdeutschen Zeitungsverleger-Verein, 1. Aufl., Bielefeld 1947, S. 244.

8. Nach Akten des "Verein Union-Presse ${ }^{\alpha}$, Soest/Hagen/Bonn.

9. Artur Sträter: Wir beginnen, in: ${ }_{n}$ Westfalenpost $^{\star}{ }^{(S o e s t)}$ ), 1 Jg. / Nr. 1 (26. April 1946).

10. N. N.: Am Anfang des Weges, in: "Westfalenpost" ${ }^{4}$ (Soest), 1. Jg. / Nr. 1 (26. April 1946).

11. Handbuch Deutsche Presse, a.a.O.

12. Eine Kurzmonographie über die "Ruhr-Nachrichten" (Dortmund) wird in absehbarer Zeit in dieser Zeitschrift erscheinen.

13. Brief von Karl Peukert, Chef vom Dienst "Westfalenpost" (Hagen) an d. Verf. vom 11. Juni 1970.

14. Georg Bitter: Zur Typologie des deutschen Zeitungswesens in der Bundesrepublik Deutschland, München 1951, S. $35 \mathrm{f}$.

15. Handbuch Deutsche Presse, 2. Ausgabe, Bielefeld 1951, S. 692 bzw. 767.

16. Die Deutsche Presse 1954, hrsgg. vom Institut für Publizistik an der Freien Universität Berlin, Berlin 1954, S. 50.

17. Brief von Karl Peukert ... vom 30. Juni 1964, S. 2 f.

18. Willy Stamm (Hrsg.): Leitfaden für Presse und Werbung 1957, Essen-Stadtwald 1957, S. 58.

19. Brief von Karl Peukert ... vom 30. Juni 1964, S. 2.

20. In eigener Sache, in: "Westfalenpost ${ }^{\star}$ (Hagen), 30./31. Dezember 1957. 
21. Die Deutsche Presse 1961, Berlin 1961, S. 35 bzw. 64.

22. IVW-Auflagen-Liste II -70, S. 46.

23. E. F. Klotzsche: Die Marktforschung als Hilfsmittel der Werbeplanung, Frankfurt a. M. o. J. (1970), S. 7 (unpag.).

24. Brief von Karl Peukert ... vom 30. Juni 1964, S. 2.

\section{S UM M A R Y}

After the "Westfalen-Blatt" (Bielefeld) the "Westfalenpost" was the second newspaper with CDU leanings which was licensed by the British Occupation Forces in Westfalen after the Second World War. It was published from April 26, 1947, first in Soest, later in Hagen - with numerous local editions. The aim of the publication was the fight against the "Revolution of Nihilism". The editions which were published in and around Dortmund carried the additional title ${ }_{n}$ Ruhr-Nachrichten ${ }^{\text {. }}$. Under this name there was published since 1949 an independent daily newspaper by the Lensing Press (Dortmund), which has a rich Catholic tradition. In 1958 the "Westfalenpost" and "Ruhr-Nachrichten" joined their editorial and advertising staffs. The presses of this modern regional newspaper for the eastern Ruhr area remain independent.

\section{R E S U M E N}

Después del „Westfalen-Blatt“ (Bielefeld), el .Westfalenpost" fue el segundo periódico de inspiración CDU que, después de la II Guerra Mundial, recibió permiso de impresión de las fuerzas de ocupación británicas. Apareció el 26 de Abril de 1947 - primero en Soest, más tarde en Hagen - en numerosas ediciones locales. Su finalidad publicística era la lucha contra la "Revolución del Nihilismo". Las ediciones de Dortmund y alrededores levaban el subtîtulo "Ruhr-Nachrichten“. Bajo este nombre se publica, desde 1949, un diario independiente en la tradicionalmente católica Editorial Lensing (Dortmund). En 1958, el "Westfalenpost“ y el „Ruhr-Nachrichten" establecieron una comunidad de trabajo en lo relacionado con el aspecto de redacción y publicidad. Las Editoriales de estos modernos periódicos regionales de la parte oriental del territorio del Ruhr permanecieron siendo independientes. 